\title{
The ARCHES Space-Analogue Demonstration Mission: Towards Heterogeneous Teams of Autonomous Robots for Collaborative Scientific Sampling in Planetary Exploration
}

\author{
Martin J. Schuster ${ }^{1}$, Marcus G. Müller ${ }^{1}$, Sebastian G. Brunner ${ }^{1}$, Hannah Lehner ${ }^{1}$, Peter Lehner ${ }^{1}$, Ryo Sakagami ${ }^{1}$, \\ Andreas Dömel ${ }^{1}$, Lukas Meyer ${ }^{1}$, Bernhard Vodermayer ${ }^{1}$, Riccardo Giubilato ${ }^{1}$, Mallikarjuna Vayugundla ${ }^{1}$, \\ Josef Reill $^{1}$, Florian Steidle ${ }^{1}$, Ingo von Bargen ${ }^{1}$, Kristin Bussmann ${ }^{1}$, Rico Belder ${ }^{1}$, Philipp Lutz ${ }^{1}$, \\ Wolfgang Stürzl ${ }^{1}$, Michal Smíšek ${ }^{1}$, Moritz Maier ${ }^{1}$, Samantha Stoneman ${ }^{1}$, Andre Fonseca Prince ${ }^{1}$, \\ Bernhard Rebele ${ }^{1}$, Maximilian Durner ${ }^{1}$, Emanuel Staudinger ${ }^{2}$, Siwei Zhang $^{2}$, Robert Pöhlmann ${ }^{2}$, \\ Esther Bischoff ${ }^{3}$, Christian Braun ${ }^{3}$, Susanne Schröder ${ }^{4}$, Enrico Dietz ${ }^{4}$, Sven Frohmann ${ }^{4}$, Anko Börner ${ }^{4}$, \\ Heinz-Wilhelm Hübers ${ }^{4}$, Bernard Foing ${ }^{5}$, Rudolph Triebel ${ }^{1}$, Alin O. Albu-Schäffer ${ }^{1}$, and Armin Wedler ${ }^{1}$
}

\begin{abstract}
Teams of mobile robots will play a crucial role in future missions to explore the surfaces of extraterrestrial bodies. Setting up infrastructure and taking scientific samples are expensive tasks when operating in distant, challenging, and unknown environments. In contrast to current single-robot space missions, future heterogeneous robotic teams will increase efficiency via enhanced autonomy and parallelization, improve robustness via functional redundancy, as well as benefit from complementary capabilities of the individual robots. In this article, we present our heterogeneous robotic team, consisting of flying and driving robots that we plan to deploy on scientific sampling demonstration missions at a Moon-analogue site on Mt. Etna, Sicily, Italy in 2021 as part of the ARCHES project. We describe the robots' individual capabilities and their roles in two mission scenarios. We then present components and experiments on important tasks therein: automated task planning, high-level mission control, spectral rock analysis, radio-based localization, collaborative multi-robot 6D SLAM in Moon-analogue and Marslike scenarios, and demonstrations of autonomous sample return.
\end{abstract}

Index Terms-Space Robotics and Automation; Multi-Robot Systems; Autonomous Agents

Manuscript received: February, 24, 2020; Revised May 19, 2020; Accepted June 16, 2020.

This paper was recommended for publication by Editor Jonathan Roberts upon evaluation of the Associate Editor and Reviewers' comments. This work was supported by the Helmholtz Association, project alliance ROBEX (contract number HA-304) and project ARCHES (contract number ZT-0033).

${ }^{1}$ M. J. Schuster, M. G. Müller, S. G. Brunner, H. Lehner, P. Lehner, R. Sakagami, A. Dömel, L. Meyer, B. Vodermayer, R. Giubilato, M. Vayugundla, J. Reill, F. Steidle, I. von Bargen, K. Bussmann, R. Belder, P. Lutz, W. Stürzl, M. Smíšek, M. Maier, S. Stoneman, A. Fonseca Prince, B. Rebele, M. Durner, R. Triebel, A. O. Albu-Schäffer, and A. Wedler are with the Inst. of Robotics and Mechatronics, German Aerospace Center (DLR), Germany \{first.lastname@dlr.de\}

${ }^{2}$ E. Staudinger, S. Zhang, and R. Pöhlmann are with the Inst. of Communications and Navigation, DLR, Germany \{first. lastname@edlr.de\}

${ }^{3} \mathrm{E}$. Bischoff and C. Braun are with the Inst. of Control Systems, Karlsruhe Institute of Technology (KIT), Germany \{first. lastname@@kit.edu\}

${ }^{4}$ S. Schröder, E. Dietz, S. Frohmann, A. Börner, and H-W. Hübers are with the Inst. of Optical Sensor Systems, DLR, Germany ffirst.lastnamededlr.de\}

${ }^{5} \mathrm{~B}$. Foing is with the European Space Research and Technology Centre (ESTEC) \& International Lunar Exploration Working Group EuroMoonMars, European Space Agency (ESA), Netherlands \{Bernard.Foinglesa.int

Digital Object Identifier (DOI): 10.1109/LRA.2020.3007468

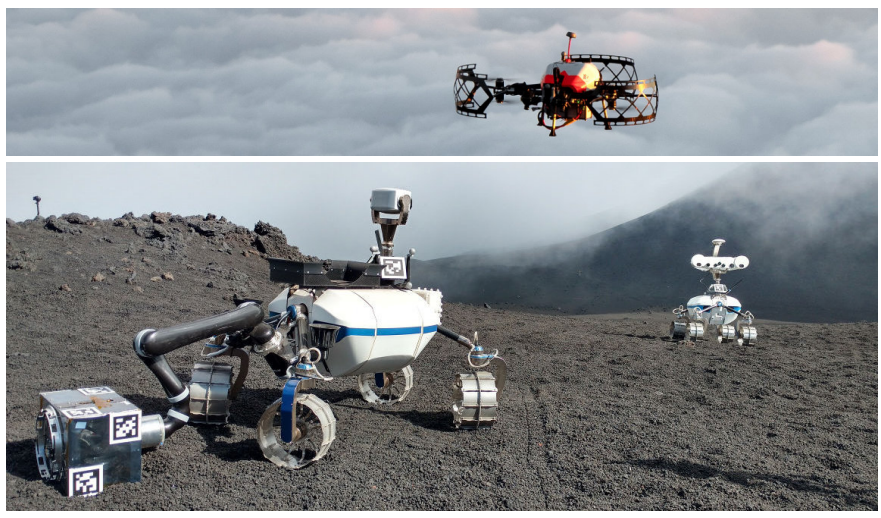

Fig. 1: Our Lightweight Rover Units (LRUs) [5] and drone ARDEA [7] test their skills for autonomous navigation, exploration, and sampling at a Moon-analogue site on Mt. Etna.

\section{INTRODUCTION}

$\mathbf{T}$ EAMS of mobile robots that operate on the surfaces of extraterrestrial bodies will play a crucial role in future space exploration endeavors [1], [2]. Compared to current single-robot missions, they will improve efficiency via parallelization and robustness via functional redundancy. In addition, heterogeneous teams benefit from complementary capabilities of their individual agents [3], [4]. However, several key tasks in lunar and planetary exploration missions, such as infrastructure installation and scientific sampling, remain challenging: robots operating in Global Navigation Satellite System (GNSS)-denied environments have to rely on spacesuitable on-board sensors to navigate, explore, and sample unknown areas in rough terrain. Long round-trip times, ranging from seconds (Moon) to many minutes (Mars), render direct teleoperation impractical to impossible. Thus, local robot autonomy is required as the basis for any coordinated joint action within the team. For this, the robots need to localize themselves online, map their surroundings, and share information about the environment and their position therein. 
In the ARCHES project by the Helmholtz Association, we develop heterogeneous, autonomous, and interconnected robotic systems and technologies to tackle these challenges [8]. We will show their capabilities during demonstration missions in the summer of 2021 at a Moon-analogue site on the volcano Mt. Etna on Sicily, Italy. ${ }^{1}$ While in ROBEX [9], a single robot deployed a seismic sensor array, we focus in ARCHES on the cooperation in a heterogeneous team. Our robotic team includes a drone for fast scouting and rovers with scientific instruments and manipulation capabilities for infrastructure installation, close-up analyses, and sample return. In Fig. 1, we give an impression of our preliminary experiments at the test site conducted in 2017 and 2019. In this article, we present

- our heterogeneous robotic team and its capabilities

- two mission scenarios: the setup of a distributed radio telescope as well as geological exploration and sampling

- experiments testing key components for the missions

It is in parts based on our IROS 2019 workshop paper [10]. In addition to extended descriptions of central aspects, we present novel contributions going beyond the workshop:

- mission scenario for the installation of a low-frequency radio array (LOFAR) and a radio-based localization of the array elements via our novel Radio-CPT system

- high-level mission control with automated task planning, multi-robot monitoring and coordination, autonomous task execution, and shared robot autonomy

- rock and sand analysis via laser-induced breakdown spectroscopy (LIBS) to be conducted by our rover

- collaborative simultaneous localization and mapping (SLAM) with three agents at the Moon-analogue site

We showed more than 35 live runs of collaborative SLAM and autonomous sample-return missions in a Mars-like scenario at the International Astronautical Congress (IAC) 2018, the world's largest annual gathering of space professionals with more than 6500 participants. Accompanying this article, we provide a video thereof as well as of our novel three-agent 6D SLAM experiment at a Moon-analogue site on Mt. Etna.

\section{RELATED WORK}

In this section, we discuss related work on multi-robot teams for planetary exploration. Focusing on scientific measurements, [11], [12] employ teleoperated rovers to carry instruments, limiting the use of vision sensors to enhance the situational awareness of the operators. LUNARES [3] demonstrated the ability of a semi-autonomous heterogeneous robotic team to explore lunar craters, artificially replicated indoors with a challenging slope and analogous lighting characteristics. In [13], a team of two hybrid wheeled/legged robots is remotely operated via an augmented reality interface to collect geological samples in a Mars-analogue environment. Assisted teleoperation is investigated in [14], where a swarm of GPS-based drones is deployed on Lanzarote to gather aerial images with the goal to minimize the cognitive load of the operators. In contrast to these approaches and to [4], our

\footnotetext{
${ }^{1}$ The ARCHES demonstration mission was originally scheduled for 2020 , but is now planned to be shifted to 2021 due to the COVID-19 pandemic.
}

robotic team will primarily operate in full autonomy governed by a complex mission control framework. The authors of [15] present a multi-robot coordination framework to map planetary caves. However, they employ rather simple wheeled/tracked robots evaluated in generic artificial environments. In contrast to the LIDAR-based vision in [3], [4], [13], [15], we use stereo cameras as a space-qualifiable sensor setup. Furthermore, our goal is to deploy a robot team at an outdoor Moon-analogue site to explore, deploy sensors, and perform scientific analyses.

\section{Heterogeneous Robotic Team}

Our heterogeneous robotic team consists of flying and driving robots as well as static and mobile infrastructure elements such as a lander and multi-purpose payload boxes [9], [17]. While drones on extraterrestrial bodies [2] require adaptions to their propulsion systems, e. g., realized by the Mars helicopter "Ingenuity" to be launched this summer [18], our drone allows us to implement and test transferable navigation and visual detection algorithms. We provide an overview of all systems in Table I. The robots benefit from their complementary capabilities: our drone ARDEA [6], [7] acts as a fast scout that can easily reach hard-to-access places such as craters or caves, whereas our first planetary exploration rover LRU1 [5] performs close-up inspections with its science camera and can transport ARDEA for energy efficiency reasons. Our second rover, LRU2 [5], [16] with its manipulator arm, takes soil and rock samples as well as transports, deploys, and manipulates payload boxes. The latter house scientific instruments and mobile infrastructure elements, e.g., to perform elemental analyses of rocks or to extend the robots' communication range. The lander acts as a base station and defines an onsite coordinate frame as a global landmark for all robots.

\section{ScIENTIFIC Exploration ScEnARios}

\section{A. Low-Frequency Radio Array (LOFAR)}

Setting up radio-astronomical instruments such as LOFARs on the far-side of the Moon allows probing the universe to unprecedented depth, as the Moon has no dense ionosphere and its far-side is one of the most radio-quiet areas in the inner solar system [19]. In this ARCHES scenario, we demonstrate key technologies to install, operate, and maintain a distributed
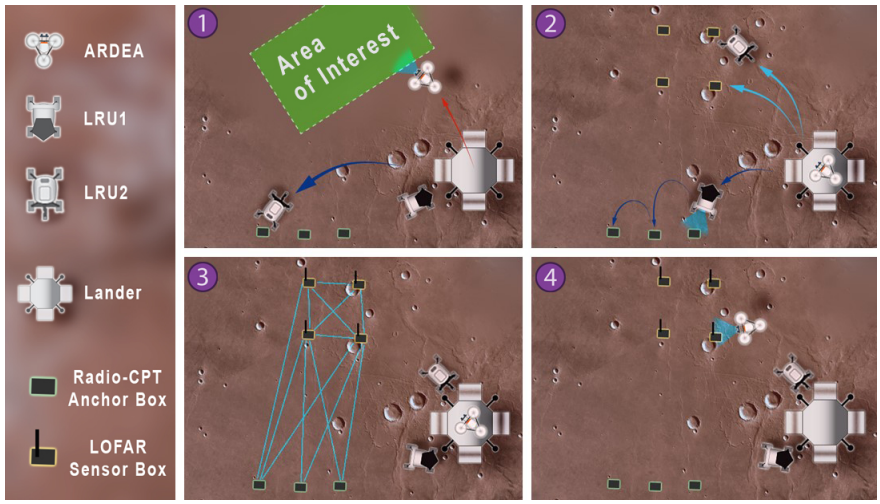

Fig. 2: ARCHES scenario with collaborative installation and maintenance of a low-frequency radio array (LOFAR). 


\begin{tabular}{|c|c|c|}
\hline System & System Features & Capabilities \\
\hline ARDEA [6] & $\begin{array}{l}\text { - Flying system with high maneuverability } \\
\text { - Ultra wide-angle stereo }\left(240^{\circ} \text { vertical, } 80^{\circ} \text { horizontal FoV) }\right. \\
\text { - Intel i7 dualcore at } 3.0 \mathrm{GHz} \text { (main computer), BeagleBone } \\
\text { ARM (control), Spartan } 6 \mathrm{LX} 75 \text { FPGA (dense stereo at } 8 \mathrm{~Hz})\end{array}$ & $\begin{array}{l}\text { - Fast autonomous scouting for exploration and mapping } \\
\text { - Identification of points of interest via semantic analyses } \\
\text { - Act as mobile radio communication relay } \\
\text { - Outdoor flight time of up to } 10 \text { min ( } 104 \mathrm{Wh} \text { battery) }\end{array}$ \\
\hline LRU1 & $\begin{array}{l}\text { - Individually controlled \& powered wheels on actuated bogies } \\
\text { - Pan/tilt camera head with stereo navigation cameras } \\
\text { - Scientific cameras: infrared camera, narrow-angle camera and } \\
\text { stereo cameras with spectral filter wheels } \\
\text { - Take-off and landing platform for ARDEA } \\
\text { - Intel i7 quadcore at } 2.7 \mathrm{GHz} \text { (main computer), Intel Atom } \\
\text { (control), Spartan 6LX75 FPGA (dense stereo at } 11 \mathrm{~Hz} \text { ) }\end{array}$ & $\begin{array}{l}\text { - Autonomous exploration and mapping in rough terrain } \\
\text { - Carrier for ARDEA } \\
\text { - Scientific analyses of rocks etc. with spectral and ther- } \\
\text { mal cameras } \\
\text { - Autonomous operation time of up to } 1.5 \mathrm{~h} \text { in outdoor } \\
\text { space-analogue environment }(2 \times 208 \mathrm{Wh} \text { batteries })\end{array}$ \\
\hline LRU2 & $\begin{array}{l}\text { - Individually controlled \& powered wheels on actuated bogies } \\
\text { - Pan/tilt camera head with stereo navigation cameras } \\
\text { - Manipulator arm with automatic docking interface } \\
\text { - Carriers for payload boxes (see below) and exchangeable tools } \\
\text { (robotic hand to grasp stones \& shovel to sample soil) } \\
\text { - Intel i7 quadcore at } 2.7 \mathrm{GHz} \text { (main computer), Intel Atom } \\
\text { (control), Spartan 6LX75 FPGA (dense stereo at } 15 \mathrm{~Hz} \text { ) }\end{array}$ & $\begin{array}{l}\text { - Autonomous exploration and mapping in rough terrain } \\
\text { - Collection of geological samples (sand or small rocks) } \\
\text { - Deployment of scientific instruments (e.g., radio anten- } \\
\text { nas or mobile spectrometers) } \\
\text { - Deployment of infrastructure (e.g., radio repeaters) } \\
\text { - Autonomous operation time of up to } 1.5 \mathrm{~h} \text { in outdoor } \\
\text { space-analogue environment }(2 \times 208 \mathrm{Wh} \text { batteries) }\end{array}$ \\
\hline Lander & $\begin{array}{l}\text { - Solar panels and batteries } \\
\text { - Computational power } \\
\text { - Communication link to mission control on Earth } \\
\text { - Storage for payload boxes with sample containers, scientific } \\
\text { instruments, and infrastructure elements }\end{array}$ & $\begin{array}{l}\text { - Perform computationally expensive tasks } \\
\text { - Save and aggregate data for downlink to Earth } \\
\text { - Gather solar power and recharge robots } \\
\text { - Store samples for future analysis } \\
\text { - Act as artificial landmark for navigation }\end{array}$ \\
\hline $\begin{array}{c}\text { Payload Boxes } \\
{[9],[17]}\end{array}$ & $\begin{array}{l}\text { Variants of payload box content: } \\
\text { - Scientific instruments: } \\
\text { - Low frequency radio array (LOFAR) antenna elements } \\
\text { - Laser-induced breakdown spectroscopy (LIBS) } \\
\text { - Radio-based positioning system (Radio-CPT) \& radio relay } \\
\text { - Soil or rock sample container } \\
\text { Each box has a common docking adapter to be grasped by LRU2. }\end{array}$ & $\begin{array}{l}\text { Depending on content and placement of box: } \\
\text { - Take scientific measurements } \\
\text { - Analyze elemental rock or soil composition } \\
\text { - Carry multiple soil or rock samples } \\
\text { - Localize payload boxes via Radio-CPT } \\
\text { - Extend communication range } \\
\text { - Provide power and communication to instruments } \\
\text { - Act as artificial landmark for navigation }\end{array}$ \\
\hline
\end{tabular}

TABLE I: Members of our heterogeneous robotic team with their complementary implemented and planned capabilities.

LOFAR for a carrier frequency between $20 \mathrm{MHz}$ and $30 \mathrm{MHz}$ in an area of about $100 \mathrm{~m} \times 100 \mathrm{~m}$. A LOFAR antenna element in the ARCHES analogue mission consists of a sensor payload box comprising a novel radio communication, positioning, and time-synchronization (Radio-CPT) system, a power supply and house-keeping unit, and a software-defined radio with a selfunfolding short dipole to receive radio signals from space.

In the LOFAR mission sketched out in Fig. 2, a team of scientists and operators select favorable array geometries for radio-astronomy observations. This selection process is assisted through a performance prediction software, taking into account resulting array directivity, wireless communication range, and Radio-CPT based self-localization accuracy. ARDEA then explores the area where sensor boxes shall be placed (1). In the meantime, LRU2 places Radio-CPT anchor boxes next to the lander, which are used for a precise localization of the antenna elements later on. A minimum of three anchors is needed to align the local LOFAR and global lander coordinate frames. Afterwards, LRU2 picks four LOFAR sensor boxes from the lander and places them in the afore defined array geometry, while LRU1 uses its cameras to measure in the Radio-CPT anchors locations w.r.t. the lander (2). At least three LOFAR boxes are required to determine the low frequency signal's direction of arrival (azimuth and elevation), while a larger number of boxes will allow an increased array aperture. For this, our system can be scaled up in a straightforward fashion. After placement, our RadioCPT system automatically locates all boxes precisely: an

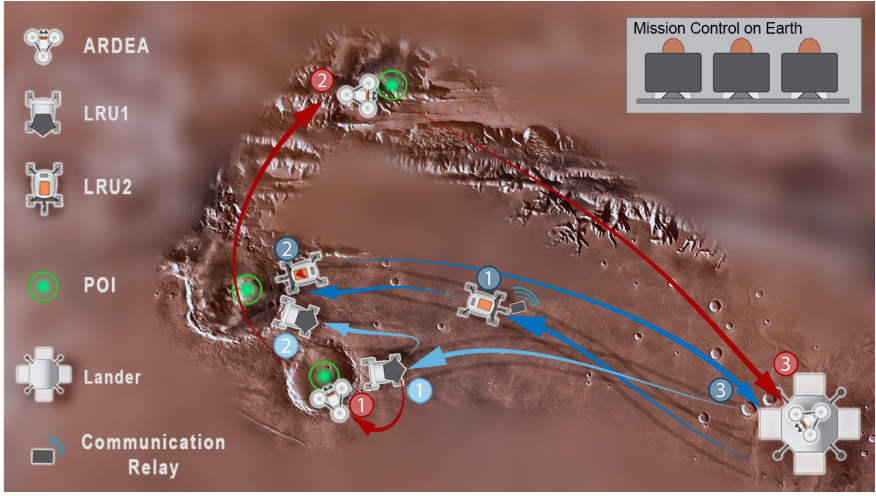

Fig. 3: ARCHES scenario with collaborative geological exploration and sampling (numbers indicate sequence of tasks).

important prerequisite to operate a distributed array. Short dipole antennas in each sensor box are released, the LOFAR is in-field calibrated, and the LOFAR signal reception starts (3). Pre-processed received signals are sent back to mission control for further processing and visualization by scientists, e. g., to identify S-type or L-type Jovian radio bursts [20]. In addition, scientists can select different modes of operation in postprocessing, i. e., monitoring solar radio-bursts during daytime, estimating the angle of arrival of a strong source, or digitally steering the LOFAR beam in a specific direction. After disruptive events such as a dust storm on Mars, ARDEA can be sent to visually inspect the antenna array for damages (4). 


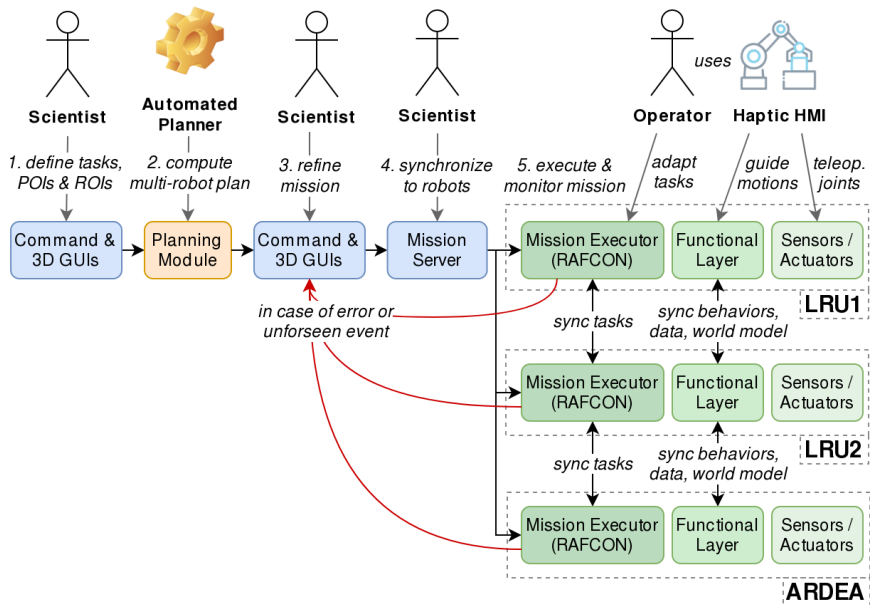

Fig. 4: Overview of our high-level mission control flow.

\section{B. Geological Exploration and Sampling}

The goal of the upcoming ARCHES geological sampling demonstration mission on Mt. Etna in 2021 is the creation of an accurate map of a target region, the spectral analysis of rocks, as well as the collection of sand and rock samples. For this, we will leverage the complementary capabilities of our heterogeneous team of (semi-)autonomous robots. In Fig. 3, we give an overview of the mission scenario. It involves the deployment of our robot systems and infrastructure elements listed in Table I. A landing spot had been selected by a team of scientists and mission operators based on prior knowledge from satellite imagery. It is located near three geologically interesting locations marked as points of interest (POIs) in Fig. 3. As a first step after landing, our rover LRU1 (@) is sent out to explore the closest POI in a nearby crater. It drives towards the target area while autonomously avoiding obstacles on its way. ARDEA, our drone, is carried by the rover on its transport and take-off platform. Upon reaching the target area, the 3D map created by LRU1 shows that the crater slope is too steep for the rover to enter. Therefore, mission control decides to launch the drone ARDEA (1) to further explore and map the crater from inside. At the same time, LRU2 (1) is sent out to place a payload box with a communication relay in between the lander and the two other, farther-away POIs to ensure radio coverage. In a second step, LRU1 (2) moves towards the next nearby, easier accessible, POI and uses its spectral cameras to identify stones for further inspection. Thus, mission control sends LRU2 (2) to deploy its LIBS instrument for laser spectroscopy and to collect the most promising rock sample, which it then returns to the lander (3). In the meantime, ARDEA (2) inspects and maps the final POI with its ultra-wide angle cameras to estimate the scientific gain of close-up inspection with the other robots. It then returns to the lander (3) for recharging.

\section{High-LEVEL Mission CONTROL}

For high-level mission control, we developed a framework for the efficient operation of multiple heterogeneous robots without requiring advanced robotics skills. The overall flow, see Fig. 4, consists of (1) task definition, (2) automated initial

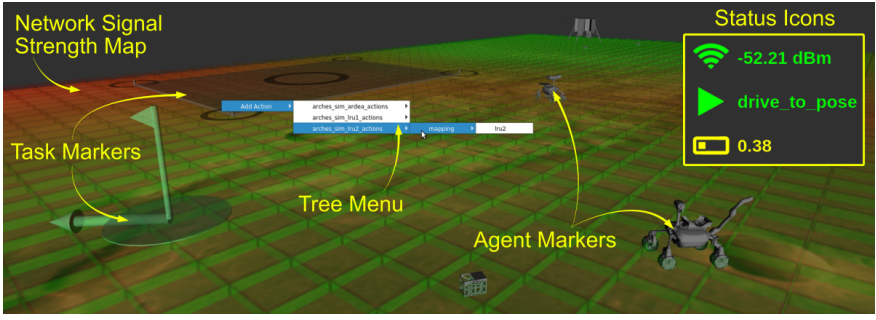

Fig. 5: Screenshot of the 3D GUI and status icons (per robot) of the ROSMC high-level mission control framework.

mission planning, (3) mission refinement, (4) synchronization to the robots, and (5) mission execution and monitoring.

To generate an initial mission plan, we use an automated planning algorithm that can handle cooperative tasks and precedence constraints relevant in both ARCHES missions. First, a greedy initialization similar to [21] provides a feasible initial solution. It is then refined via an optimization heuristic based on the idea of a relocation neighborhood known in vehicle routing [22], which we adapted to multi-robot taskallocation. For details and evaluations of the coordination algorithm, we refer to [23]. The tasks to be scheduled for the individual robots belong to predefined types. For ARCHES, the main ones include "take image" (ARDEA, LRU1, LRU2), "take science image" (LRU1), "take sample" (LRU2), and "take sample scientific mode" (LRU2 takes sample based on spectral images by LRU1). In addition, there are auxiliary task types for LRU2: "install radio range extender", "pick payload box", and "place payload box". Each task is related to a POI for its execution. While main tasks are specified by the scientist, auxiliary tasks are added automatically whenever needed. Each auxiliary task adds a precedence constraint as, e.g., a sample container needs to be fetched prior to collecting a sample. The planner calculates an individual schedule for each robot, optimizing the overall mission time, driven distances, and energy consumption.

To enable intuitive task definition, mission refinement, and robust, distributed mission execution and monitoring, we introduce our novel ROSMC (ROS Mission Control) framework. It provides two graphical user interfaces (GUIs): a 3D GUI visualizing the global map, the agents' poses, status, POIs, and ROIs (see Fig. 5) as well as a command GUI to define task sequences and parameterization. Mission control can adapt the pre-planned schedule of each robot: the 3D GUI allows to add tasks via a tree menu and to modify their POIs or ROIs, the command GUI to rearrange their order. The entire mission consists of a synchronized and a non-synchronized part, both being stored and tracked on a central mission server with low computational requirements. The former part, which is read-only in the GUIs, is sent to each robot so that they can execute the mission on board. The mission progress is stored locally and sent to the server when communication becomes available. In case of an error during runtime, the synchronized part can be deleted from the robot, adapted, and then re-synchronized. Powerful execution modalities based on hierarchical state machines modeled in our open source framework RAFCON [24] allow to pause and resume the 

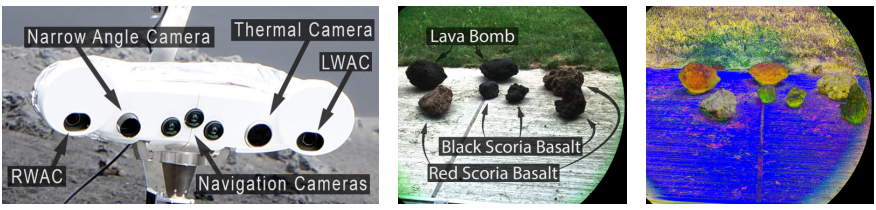

(a) ScienceCam sensor head (b) RGB image and science product with its seven cameras

of volcanic rocks

Fig. 6: ScienceCam (a) and science product (b) visualized as $R G B=\left(G e o 4, \frac{G e o l-B l u e}{G e o l+B l u e}, \frac{G e o 6-G e o l}{G e o 6+G e o l}\right)$. Whereas the lava bomb and black scoria basalt look similar in the RGB image, they can be clearly distinguished in the science product.

behavior of each robot, to modify it, and to exchange the robots' entire command sequences during their mission. Task synchronization is achieved peer-to-peer without the server: robots communicate task ids with each other to coordinate inter-robot task dependencies. We are in preparation of a separate publication with details on ROSMC and intend to release it as open source in the future.

During mission execution, control can be switched to manual mode in case teleoperation is feasible and required. We employ a robotic arm as a generic haptic human-machine interface (HMI) to teleoperate different types of robots in a heterogeneous team. Its six degrees of freedom $(\mathrm{DoF})$ allow for a direct mapping of the HMI states to the positions and orientations of the teleoperated robots, enabling an intuitive operation both in large-scale and precision tasks. Each DoF is actuated, which permits a continuous shift of the optionbased levels of automation [25], covering the whole range from manual control via shared autonomy to full autonomy. Thus, operators can be supported with a cooperating automation to reduce their workload during teleoperation. As the haptic feedback is computed on the local HMI controller, the overall system is robust to communication delays.

These features render our mission control software more powerful than many approaches of related work, e.g., IMPACT [26], which does not allow a parameterization of reactive behavior, and M2PEM [27], which neither supports heterogeneous teams nor allows to switch to manual control.

\section{SPectral ANAlysis of Rock SAMPLeS}

The geological exploration of planetary surfaces aims at understanding the history of our solar system and supports the search for signs of life, resources, and habitable areas.

\section{A. ScienceCam}

LRU1 is equipped with a scientific camera, shown in Fig. 6 and introduced in [9] as ScienceCam. It has a concept similar to the PanCam of the ExoMars rover [28]. In addition to three navigation cameras, it houses wide-angle stereo (WAC, Manta G-145B NIR), a thermal (Xenics Serval-640), and a narrowangle camera (Manta G-505C). The two WACs have spectral filter wheels, each containing three color filters $(100 \mathrm{~nm}$ bandwidth) and six narrow band (10 nm bandwidth) geology filters (Geol-Geo12) across ranges of $440-660 \mathrm{~nm}$ and $720-1000 \mathrm{~nm}$. By combining several bands, science products

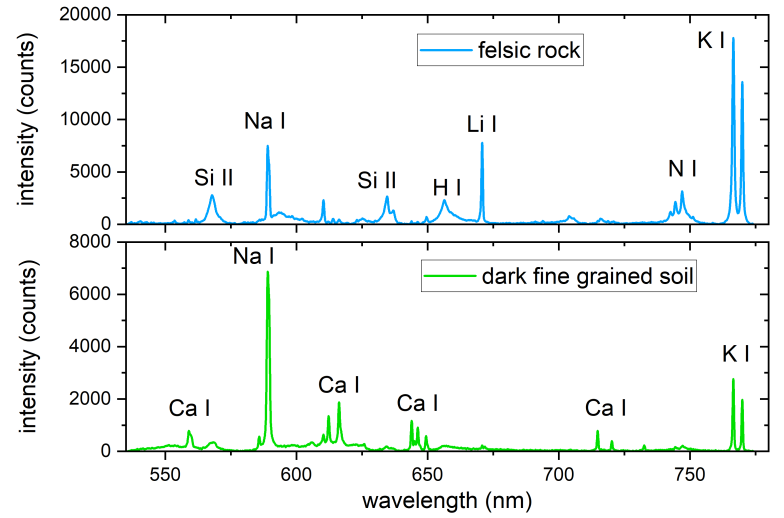

Fig. 7: LIBS spectra of a felsic rock and a dark fine-grained soil obtained with the ARCHES LIBS module for the LRU. The continuous emission was removed by a polynomial fit.

can be calculated to distinguish different types of rocks. In Fig. 6(b), we give an example based on a solely geometrically calibrated camera with the Blue $(460 \mathrm{~nm})$, Geol $(440 \mathrm{~nm})$, Geo4 $(560 \mathrm{~nm})$, and Geo6 $(660 \mathrm{~nm})$ filters. For examination, we visualized the resulting channels as RGB: rocks that cannot be discriminated in the visible spectrum clearly stand out.

\section{B. Laser-Induced Breakdown Spectroscopy (LIBS)}

To perform elemental analysis of rocks and soil in its environment, LRU2 can equip itself with a laser-induced breakdown spectroscopy (LIBS) instrument that is housed in a payload box. LIBS can be used in close-up or remote configurations and gains more and more relevance for planetary in-situ exploration. Our LIBS module is connected to the rover's arm and built from commercial components (laser, spectrometer, micro-controller). It weighs about $1 \mathrm{~kg}$ and scans a small surface in close distance from the modular box with sampling rates of up to $30 \mathrm{~Hz}$. A Nd:YAG laser with $8 \mathrm{~mJ}$ and $6 \mathrm{~ns}$ pulses at $1064 \mathrm{~nm}$ is used to create the micro plasma. The spectrometer covers a range of wavelengths of $550-770 \mathrm{~nm}$, in which major rock-forming elements such as silicon, calcium, sodium, and potassium can be detected, see Fig. 7. Also minor and trace elements such as hydrogen and lithium can be observed, as well as the emission of nitrogen, which results from a breakdown of the ambient atmosphere.

\section{Distributed Collaborative Multi-Robot 6D SLAM IN MOON-ANALOGUE ENVIRONMENT}

Multi-robot 6D Simultaneous Localization and Mapping (SLAM) allows robots to localize themselves w. r. t. each other and build a shared dense 3D model of their environment for autonomous exploration, mission planning, and the coordination of joint actions. We employ our online and on-board collaborative multi-robot SLAM framework [29], [30] to map roughterrain environments in a distributed fashion: high-frequency and high-bandwidth sensor data is locally aggregated in realtime on each robot and then exchanged via so-called submaps between the systems. In contrast to other recent distributed SLAM approaches such as [31], [32], ours does not rely on a central server to delegate optimization and map managemnet 


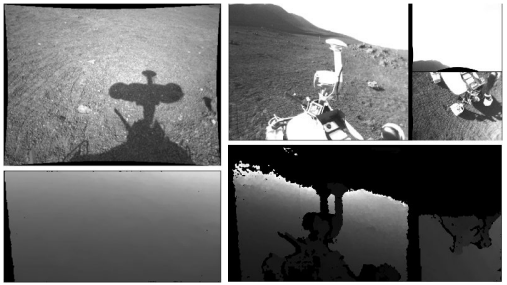

(a) LRU1 narrow-angle and ARDEA ultra wide-angle camera \& depth data

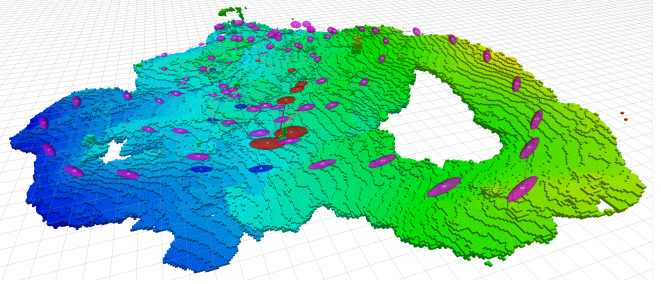

(b) Height-colored probabilistic voxel-grid map by three agents (LRU1, LRU2, ARDEA sensor stack)

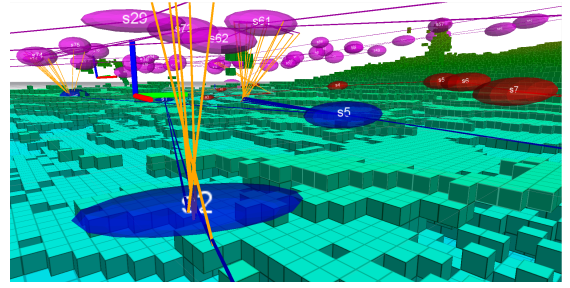

(c) Detail of (b): robot detections (orange edges) between ARDEA and both rovers

Fig. 8: Distributed multi-robot 6D SLAM in challenging conditions (harsh shadows, overexposures due to bright sunlight); data acquired in three-agent experiment at Moon-analogue site on Mt. Etna $\left(10 \mathrm{~cm}\right.$ map resolution, $1 \mathrm{~m}^{2}$ background grid cells); ellipsoids show submap origins and positional uncertainty for LRU1 (blue), LRU2 (red), ARDEA sensor stack (pink).

\begin{tabular}{r|c|c|c} 
Multi-robot SLAM graph & LRU1 & LRU2 & ARDEA \\
\hline Number of robot poses & 48 & 28 & 42 \\
\hline Number of submaps & 7 & 10 & 78 \\
\hline Number of robot detections & 0 & 17 & 42
\end{tabular}

TABLE II: Statistics for collaborative mapping exp. of Fig. 8.

tasks. Instead, every agent maintains a full SLAM graph for incremental online global optimization, exchanging constraints (submap matches and robot detections) and compacted submap data (3D voxel-grids and pointclouds) with no need for duplicate transmissions. This allows distributing the computational workload and keeping communication bandwidth requirements low. We refer to [29], [30] for further details on our SLAM framework, including more than 30 real-world experiments with five different robot platforms and evaluations regarding accuracy, runtime, and ressource requirements.

Going beyond our previous two-agent SLAM experiments [29], [30], [10], we present first three-agent SLAM results on data acquired by a heterogeneous team of two LRU rovers and the sensor stack of our drone ARDEA at the Moon-analogue site [9]. In Fig. 8(a), we highlight challenges faced by the robots observing the world with their stereo cameras. In Fig. 8, we show the resulting dense multi-robot 3D map on a slope of Mt. Etna. Both rovers autonomously explored a given area by maximizing their expected information-gain [33]. In the meantime, we carried the sensor stack of ARDEA between them, simulating a flight that explores further areas, see the outer parts of the map in Fig. 8(b). Visual markers on both rovers allowed close-range detection by each other and by ARDEA in order create inter-robot loop closures, shown as edges in the SLAM graph in Fig. 8(c). The robots thus acted as moving landmarks for each other, enabling a joint pose and map optimization. The resulting multi-robot graph has a total of only 213 nodes and 270 factors, see Table II, and thus allows for fast optimization.

\section{RADIO-BASED LOCALIZATION (RADIO-CPT)}

The LOFAR mission (Sec. IV-A) depends on precisely locating the LOFAR boxes, which will be achieved by our radio communication, positioning, and time-synchronization (RadioCPT) system. The boxes are connected by bi-directional radio frequency (RF) links with a carrier frequency of $f_{\mathrm{c}}=5.8 \mathrm{GHz}$, a bandwidth of $B_{\mathrm{c}}=25 \mathrm{MHz}$, and a self-organized time division multiple access (SO-TDMA) protocol [34]. The localiza-

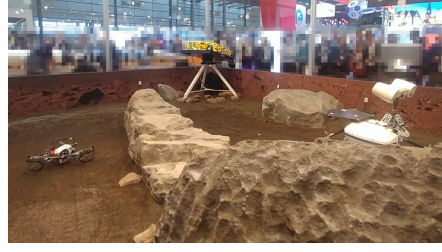

(a) Mars-like scenario with sand and large artificial rocks $\left(50 \mathrm{~m}^{2}\right)$

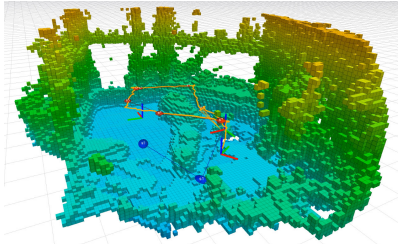

(b) Probabilistic height-colored voxel-map $(10 \mathrm{~cm}$ resolution)
Fig. 9: Multi-robot SLAM with LRU1 and ARDEA at IAC 2018; all computation online and on board both robots.

tion accuracy depends on the clock accuracy, carrier frequency, transmit power, bandwidth, and the environment, which can promote multipath propagation [35]. In the ARCHES scenario, multipath effects are, however, not significant. We developed a distributed particle filter algorithm for radio localization [36], obtaining near-optimal performance with a complexity significantly lower than other state-of-the-art algorithms like [37]. In order to deal with the instability of low-cost oscillators, we implemented a distributed Kalman filter to track their characteristics using short TDMA frames (below $100 \mathrm{~ms}$ ) [34].

We studied the joint estimation of LOFAR box location and LOFAR signal direction of arrival (DoA) in [38]. Our LOFAR setup targets signal reception at $20 \mathrm{MHz}$ carrier frequency, i.e., a wavelength of $15 \mathrm{~m}$. Accurate beam steering in azimuth and elevation requires an array localization accuracy of at least one order of magnitude higher than the carrier wavelength, which is $1.5 \mathrm{~m}$ for our system. We showed in simulation and multiple outdoor experiments [36], [34] that sub-meter accuracy can be achieved in an environment similar to the space-analogue mission, thereby verifying the feasibility of our approach. We plan to further reduce the impact of the LOFAR box location uncertainty on LOFAR DoA estimation via online phase calibration [39].

\section{INTEGRATION TESTS IN MARS-LIKE ENVIRONMENT}

As a major milestone, we integrated several components for three different multi-system live demonstrations at IAC 2018 in a Mars-like sandbox of $50 \mathrm{~m}^{2}$, shown in Fig. 9. It features a lander mockup and artificial rocks as navigational and visual obstacles. All software required for autonomous robot behavior ran online and on board the three robots.

First, we demonstrated our distributed collaborative 6D SLAM with a heterogeneous team of our rover LRU1 and 

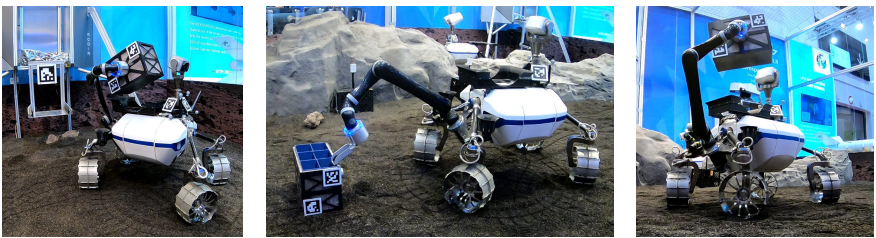

Fig. 10: Sequence of our IAC 2018 autonomous sample-return demonstration: (1) pick up container at lander, (2) extract sample, (3) reclaim container and return it to lander.

drone ARDEA in more than 35 successful live runs. Onboard and online, both robots locally created partial 3D maps, exchanged, optimized, and merged them to a collaboratively built joint map of the environment. To connect the maps via loop closure constraints, ARDEA detected visual markers on LRU1 during its flight. We show the final 3D map in Fig. 9 and refer to [30], [7] for further details on this demonstration.

Second, in further 35 live runs, we demonstrated the robust integration of our on-board navigation, task execution, and manipulation required for a fully autonomous sample-return task, as shown in Fig. 10: LRU2 retrieved a sample container, transported it to a target location, placed it on the ground, grasped a shovel, scooped a soil sample into the payload box, and returned it to the lander. For details on the method and earlier experiments on Mt. Etna, we refer to [16].

Third, we integrated our Radio-CPT system in a robotic mission scenario and demonstrated real-time radio-localization of LRU2 carrying a Radio-CPT payload box in more than 20 live runs (>30 min each). The high-rate real-time physical layer and MAC-layer processing ran in a distributed fashion in seven sensor boxes, and an off-board particle filter provided real-time $2 \mathrm{D}$ position estimates.

\section{COnclusion And Future Work}

We summarize our methods to tackle the technical core challenges of the two ARCHES missions in Table III and give an evaluation regarding their maturity and open challenges. All of our robots are equipped with space-suitable sensor setups for visual-inertial navigation in GNSS-denied environments. A fully decentralized and in large parts distributed computation allows an efficient utilization of limited resources and ensures robustness to failures of individual agents. All modules run onboard and online, i. e., at a rate sufficient for reactive behaviors, and exchange locally pre-processed data for low-bandwidth communication. This allows full on-board autonomy, which is crucial in the light of unreliable and delayed communication links. We designed our system to scale for heterogeneous teams of $n<10$ agents, thus swarms of hundreds of robots envisioned for the distant future will introduce further challenges, in particular w.r.t. data exchange. Our modular approach allows an easy adaption of core components to different robots and settings. Adding new types of sensors or robots, for example, does not require any changes to the other agents in a team as our multi-robot 6D SLAM connects them on the level of poses and aggregated (sub)map data. Furthermore, our framework RAFCON [24] (task execution) is available as open source and ROSMC (mission control) planned to be in the future.
For the ARCHES demonstration on Mt. Etna in 2021, we will bring together and extend the systems and capabilities shown at IAC 2018. As we experienced in the ROBEX campaigns [9], the additional challenges of full system integration, environment conditions (terrain, light, wind, local gravity, air density, etc. different to the lab), and logistics are not to be underestimated. Furthermore, we started to adapt individual modules for the Martian Moons eXploration mission (MMX) to navigate a rover on Phobos in 2026 [42].

\section{ACKNOWLEDGMENT}

We thank the Mobile Robots Group at DLR-RMC and the ARCHES \& ROBEX teams for support with the systems and experiments, and the reviewers for their insightful suggestions.

\section{REFERENCES}

[1] ISECG, "Global Exploration Roadmap," International Space Exploration Coordination Group, Tech. Rep., 2018.

[2] Y. Gao and S. Chien, "Review on space robotics: Toward top-level science through space exploration," Science Robotics, vol. 2, 2017.

[3] F. Cordes, et al., "LUNARES: lunar crater exploration with heterogeneous multi robot systems," Intelligent Service Robotics, vol. 4, 2011.

[4] M. Eich, et al., "Towards Coordinated Multi-Robot Missions for Lunar Sample Collection in Unknown Environment," J. of Field Robotics, vol. 31, 2014.

[5] M. J. Schuster, et al., "Towards Autonomous Planetary Exploration: The Lightweight Rover Unit (LRU), its Success in the SpaceBotCamp Challenge, and Beyond," J. of Intelligent \& Robotic Systems, 2017.

[6] M. G. Müller, et al., "Robust Visual-Inertial State Estimation with Multiple Odometries and Efficient Mapping on an MAV with UltraWide FOV Stereo Vision," in IROS, 2018.

[7] P. Lutz, et al., "ARDEA - An MAV with skills for future planetary missions," J. of Field Robotics, 2019.

[8] A. Wedler, et al., "From single autonomous robots toward cooperative robotic interactions for future planetary exploration missions," in International Astronautical Congress (IAC), 2018.

[9] — "First Results of the ROBEX Analogue Mission Campaign: Robotic Deployment of Seismic Networks for Future Lunar Missions," in International Astronautical Congress (IAC), 2017.

[10] M. J. Schuster, et al., "Towards Heterogeneous Robotic Teams for Collaborative Scientific Sampling in Lunar and Planetary Environments," in Workshop on Informed Scientific Sampling in Large-scale Outdoor Environments at IROS, 2019.

[11] L. Graham, et al., "Moon and Mars Analog Mission Activities for Mauna Kea 2012," in IEEE AeroConf, 2013.

[12] É. Dupuis, et al., "Results from the CSA's 2015 Mars analogue mission in the desert of Utah," in i-SAIRAS, 2016.

[13] R. Sonsalla, et al., "Field testing of a cooperative multi-robot sample return mission in mars analogue environment," in ASTRA, 2017.

[14] D. St-Onge, et al., "Planetary Exploration with Robot Teams: Implementing Higher Autonomy With Swarm Intelligence," IEEE Robot. Automat. Mag., 2019.

[15] A. Husain, et al., "Mapping Planetary Caves with an Autonomous, Heterogeneous Robot Team,' in IEEE AeroConf, 2013.

[16] P. Lehner, et al., "Mobile manipulation for planetary exploration," in IEEE AeroConf, 2018.

[17] G. Tsakyridis, et al., "Power system analysis and optimization of a modular experiment carrier during an analog lunar demo mission on a volcanic environment," Acta Astronautica, vol. 155, 2019.

[18] B. Balaram, et al., "Mars Helicopter Technology Demonstrator," 2018.

[19] C. L. Carilli, et al., "Low frequency radio astronomy from the moon: Cosmic reionization and more," Cambridge University Press, 2007.

[20] P. Zarka, "Auroral radio emissions at the outer planets: Observations and theories," Journal of Geophysical Research: Planets, 1998.

[21] Y. Zhang and L. E. Parker, "Multi-robot task scheduling," in ICRA, 2013.

[22] M. W. P. Savelsbergh, "The Vehicle Routing Problem with Time Windows: Minimizing Route Duration," ORSA J. on Computing, 1992.

[23] E. Bischoff, et al., "Multi-Robot Task Allocation and Scheduling Considering Cooperative Tasks and Precedence Constraints," 2020, (submitted, preprint on arXiv). 


\begin{tabular}{|c|c|c|c|c|c|}
\hline $\begin{array}{l}\text { Technical Core } \\
\text { Challenges }\end{array}$ & $\begin{array}{l}\text { Components \& } \\
\text { Modules }\end{array}$ & Methods & $\begin{array}{l}\text { Validation } \\
\text { Status }\end{array}$ & $\begin{array}{l}\text { Experiments \& } \\
\text { Demonstrations }\end{array}$ & Results \& Open Challenges \\
\hline $\begin{array}{c}\text { High-Level } \\
\text { Mission Control }\end{array}$ & ROSMC (Sec. V) & $\begin{array}{l}\text { GUIs (in RViz + } \\
\text { RQt) \& data server }\end{array}$ & Simulation & $\begin{array}{l}\text { User study: } 9 \text { people, } \\
\text { three-robot simulations }\end{array}$ & $\begin{array}{l}\text { Concepts for sub-groups and } n>3 \\
\text { robots; will require UI adaptions }\end{array}$ \\
\hline $\begin{array}{l}\text { Automated Task } \\
\text { Planning }\end{array}$ & $\begin{array}{l}\text { Multi-robot } \\
\text { scheduling } \\
\text { (Sec. V) }\end{array}$ & $\begin{array}{l}\text { Greedy init. \& local } \\
\text { improvement } \\
\text { heuristic [23] }\end{array}$ & Simulation & $\begin{array}{l}\text { Evaluated on } 600 \text { instances } \\
\text { (up to } 3 \text { robots, } 15 \text { tasks } \\
\text { with } 9 \text { coop. tasks) [23] }\end{array}$ & $\begin{array}{l}\text { Reliable generation of feasible opti- } \\
\text { mized plans; future work: integration } \\
\text { with mission control }\end{array}$ \\
\hline $\begin{array}{l}\text { Autonomous } \\
\text { Task Execution }\end{array}$ & $\begin{array}{c}\text { RAFCON } \\
\text { (Sec. V, [24]) }\end{array}$ & $\begin{array}{c}\text { Hierarchical } \\
\text { statemachines with } \\
\text { concurrent execution }\end{array}$ & $\begin{array}{l}\text { Space-like \& } \\
\text { analogue } \\
\text { environments }\end{array}$ & $\begin{array}{l}\text { Sim. \& lab, SpaceBot- } \\
\text { Camp 2015 [5], ROBEX } \\
2017 \text { [16], IAC } 2018\end{array}$ & $\begin{array}{l}\text { Statemachines with } 8 \text { hierarchy lev- } \\
\text { els and }>700 \text { states per robot; fu- } \\
\text { ture work: multi-robot world model }\end{array}$ \\
\hline $\begin{array}{c}\text { Shared } \\
\text { Autonomy in } \\
\text { Teleoperation }\end{array}$ & $\begin{array}{l}\text { Haptic 6DoF } \\
\text { HMI (Sec. V) }\end{array}$ & $\begin{array}{l}\text { Haptic shared } \\
\text { control }[25]\end{array}$ & $\begin{array}{l}\text { Simulation \& } \\
\text { laboratory }\end{array}$ & $\begin{array}{l}\text { Teleop. of LRU camera } \\
\text { head, simulated drone, and } \\
\text { 3DoF robotic arm }\end{array}$ & $\begin{array}{l}\text { Results: } 6 \mathrm{DoF} \text { HMI with generic } \\
\text { haptics \& supporting automations; } \\
\text { pending: user study on haptic HMI }\end{array}$ \\
\hline $\begin{array}{l}\text { Fast Scouting of } \\
\text { Points of } \\
\text { Interest }\end{array}$ & $\begin{array}{c}\text { ARDEA } \\
\text { wide-angle vision } \\
\text { system (Sec.IX) }\end{array}$ & $\begin{array}{l}\text { On-board robust } \\
\text { dense stereo } \\
\text { vision [6], [7] }\end{array}$ & $\begin{array}{l}\text { Space-like \& } \\
\text { analogue } \\
\text { environments }\end{array}$ & $\begin{array}{l}\text { Laboratory [6], IAC } 2018 \\
\text { [7], Autonomous waypoint } \\
\text { flight on Mt. Etna } 2019\end{array}$ & $\begin{array}{l}\text { Successful takeoff from rover; land- } \\
\text { ing on LRU challenging due to its } \\
\text { camera mast (work in progress) }\end{array}$ \\
\hline $\begin{array}{l}\text { Dense 3D } \\
\text { Multi-Robot } \\
\text { Mapping }\end{array}$ & $\begin{array}{c}\text { 6D Multi- } \\
\text { Robot SLAM } \\
\text { Framework } \\
\text { (Sec. VII \& IX) }\end{array}$ & $\begin{array}{l}\text { Dense submaps, } \\
\text { real-time filter }+ \text { inc. } \\
\text { graph optimization } \\
{[29],[30]}\end{array}$ & $\begin{array}{l}\text { Space-like \& } \\
\text { analogue } \\
\text { environments }\end{array}$ & $\begin{array}{c}\text { Sim. \& lab., SpaceBot- } \\
\text { Camp 2015, ROBEX 2017, } \\
\text { IAC 2018 }(n>35 \text { exp. }) \\
\text { [5], [29], [30], [7] }\end{array}$ & $\begin{array}{l}\text { Evaluated with two (on-board) \& } \\
\text { three (on datasets) agents; Open } \\
\text { challenges: long-range mapping \& } \\
\text { multi-session relocalization [40] }\end{array}$ \\
\hline $\begin{array}{l}\text { Waypoint } \\
\text { Navigation \& } \\
\text { Obstacle } \\
\text { Avoidance }\end{array}$ & $\begin{array}{c}\text { Obstacle } \\
\text { mapping \& } \\
\text { 2D path planning } \\
\text { (Sec. VII \& IX) }\end{array}$ & $\begin{array}{c}\text { Stereo-adaptive } \\
\text { terrain classification } \\
\text { [30] \& adapted ROS } \\
\text { nav. stack }\end{array}$ & $\begin{array}{c}\text { Space-like \& } \\
\text { analogue } \\
\text { environments }\end{array}$ & $\begin{array}{c}\text { Sim. \& lab, SpaceBotCamp } \\
2015 \text { (LRU), ROBEX } 2016 \\
\& 2017 \text { (LRUs), IAC } 2018 \\
\text { [5], [30], [7] }\end{array}$ & $\begin{array}{l}\text { Validated on LRU rovers; ARDEA: } \\
\text { waypoint navigation \& terrain fol- } \\
\text { lowing [41]; future work: full 3D } \\
\text { path planning for ARDEA }\end{array}$ \\
\hline $\begin{array}{c}\text { Autonomous } \\
\text { Exploration }\end{array}$ & $\begin{array}{l}\text { Frontier } \\
\text { exploration } \\
\text { (Sec. VII) }\end{array}$ & $\begin{array}{l}\text { Information gain- } \\
\text { based expl. \& active } \\
\text { loop closing [33] }\end{array}$ & $\begin{array}{c}\text { Space-like \& } \\
\text { analogue } \\
\text { environments }\end{array}$ & $\begin{array}{l}\text { Sim. (40 exp.), lab, outdoor } \\
\text { \& ROBEX 2017 }(n>30 \\
\text { real exp.) [33], [5], [30] }\end{array}$ & $\begin{array}{l}\text { Tested single- and multi-robot [30] } \\
\text { (rovers); future work: full 3D expl. } \\
\text { for drone \& multi-criteria decisions }\end{array}$ \\
\hline $\begin{array}{c}\text { Payload } \\
\text { Transport \& } \\
\text { Manipulation }\end{array}$ & $\begin{array}{l}\text { Compliant } \\
\text { control, motion } \\
\text { planning, world } \\
\text { model (Sec. IX) }\end{array}$ & $\begin{array}{l}\text { Impedance control, } \\
\text { sampling-based } \\
\text { planning, } \\
\text { synchronized graph } \\
\text { database [16] }\end{array}$ & $\begin{array}{l}\text { Space-like \& } \\
\text { analogue } \\
\text { environments }\end{array}$ & $\begin{array}{c}\text { Simulation \& laboratory, } \\
\text { SpaceBot-Camp } 2015 \text { [5], } \\
\text { ROBEX } 2017 \text { [16], } \\
\text { IAC } 2018(n>35 \text { exp. })\end{array}$ & $\begin{array}{l}\text { Autonomous deployment \& pickup } \\
\text { of payload; challenges: high uncer- } \\
\text { tainties due to object detection and } \\
\text { elasticity in the rover; future work: } \\
\text { compliant whole-body control }\end{array}$ \\
\hline $\begin{array}{c}\text { LOFAR } \\
\text { Measurement }\end{array}$ & $\begin{array}{l}\text { LOFAR Payload } \\
\text { Boxes } \\
\text { (Sec. IV-A) }\end{array}$ & $\begin{array}{c}\text { Distributed } \\
\text { RF-sampling \& } \\
\text { beam steering }\end{array}$ & $\begin{array}{l}\text { Laboratory } \\
\text { setup }\end{array}$ & $\begin{array}{l}\text { Prelim. experiments: } \\
\text { single-box RF reception } \\
\text { on rooftop }\end{array}$ & $\begin{array}{l}\text { Current \& future work: integration } \\
\text { in payload boxes, proof of concept } \\
\text { experiments with multiple boxes }\end{array}$ \\
\hline $\begin{array}{l}\text { LOFAR Box } \\
\text { Localization }\end{array}$ & $\begin{array}{c}\text { Radio-CPT } \\
\text { (Sec. VIII \& IX) }\end{array}$ & $\begin{array}{l}\text { Distributed } \\
\text { particle filter [36] }\end{array}$ & $\begin{array}{c}\text { Space-like \& } \\
\text { analogue } \\
\text { environments }\end{array}$ & $\begin{array}{c}\text { Grass field } 2015 \text { [36], } \\
\text { gravel pit } 2018 \text { [34], } \\
\text { IAC 2018, Mt. Etna 2019 } \\
\end{array}$ & $\begin{array}{l}\text { Achieved sub-meter accuracy in } \\
\text { most experiments; challenges: mul- } \\
\text { tipath propagation \& instable clocks }\end{array}$ \\
\hline $\begin{array}{l}\text { Panoramic \& } \\
\text { Multi-Spectral } \\
\text { Imaging }\end{array}$ & $\begin{array}{l}\text { ScienceCam } \\
\text { (Sec. VI-A) }\end{array}$ & $\begin{array}{l}\text { Anomaly detection } \\
\text { via combination of } \\
\text { spectral images }\end{array}$ & $\begin{array}{l}\text { Lab, space- } \\
\text { analogue } \\
\text { environment }\end{array}$ & $\begin{array}{l}\text { Prelim. tests with volcanic } \\
\text { rocks [10]; panorama at } \\
\text { space-analogue site }\end{array}$ & $\begin{array}{l}\text { Radiometric \& spectral calibration } \\
\text { difficult } \rightarrow \text { differentiation but no } \\
\text { classification of minerals possible }\end{array}$ \\
\hline $\begin{array}{l}\text { Elemental Rock } \\
\text { \& Soil Analysis }\end{array}$ & $\begin{array}{c}\text { LIBS } \\
(\text { Sec. VI-B) }\end{array}$ & $\begin{array}{l}\text { Optical emission } \\
\text { spectroscopy }\end{array}$ & $\begin{array}{l}\text { Lab tests of } \\
\text { instrument }\end{array}$ & $\begin{array}{l}\text { Eight reference spectra of } \\
\text { geological samples }\end{array}$ & $\begin{array}{l}\text { Validated on rocks \& sand; integra- } \\
\text { tion with LRU payload box pending }\end{array}$ \\
\hline
\end{tabular}

TABLE III: Summary of our approaches to the core challenges of the ARCHES missions and evaluation of their maturity.

[24] S. G. Brunner, et al., "RAFCON: A Graphical Tool for Engineering Complex, Robotic Tasks," in IROS, 2016.

[25] C. A. Braun, et al., "A Continuous and Quantitative Metric for the Levels of Automation," in IFAC Symposium on Analysis Design and Evaluation of Human Machine Systems, 2019.

[26] M. Draper, et al., "Intelligent Multi-Unmanned Vehicle Planner with Adaptive Collaborative/Control Technologies (Impact)," in International Symposium on Aviation Psychology, 2017.

[27] J.-P. de la Croix, et al., "Mission modeling, planning, and execution module for teams of unmanned vehicles," in Unmanned Systems Technology XIX. International Society for Optics and Photonics, 2017.

[28] E. J. Allender, et al., "The ExoMars Spectral Tool (ExoSpec): an image analysis tool for ExoMars 2020 PanCam imagery," in SPIE Image and Signal Processing for Remote Sensing, 2018.

[29] M. J. Schuster, et al., "Distributed stereo vision-based 6d localization and mapping for multi-robot teams," J. of Field Robotics, 2018.

[30] M. J. Schuster, "Collaborative Localization and Mapping for Autonomous Planetary Exploration: Distributed Stereo Vision-Based 6D SLAM in GNSS-Denied Environments," Ph.D. dissertation, University of Bremen, 2019.

[31] K. Ebadi, et al., "LAMP: Large-Scale Autonomous Mapping and Positioning for Exploration of Perceptually-Degraded Subterranean Environments," 2020, (preprint on arXiv).

[32] M. Karrer, et al., "CVI-SLAM collaborative visual-inertial SLAM,"
Robotics and Automation Letters (RA-L), 2018.

[33] H. Lehner, et al., "Exploration with Active Loop Closing: A Trade-off between Exploration Efficiency and Map Quality," in IROS, 2017.

[34] E. Staudinger, et al., "Swarm navigation and exploration for planetary surface missions: Experimental results," in International Planetary Probe Workshop, 2019.

[35] D. Dardari, et al., "Ranging With Ultrawide Bandwidth Signals in Multipath Environments," Proc. IEEE, vol. 97, 2009.

[36] S. Zhang, et al., "Distributed direct localization suitable for dense networks," IEEE Trans. Aerosp. Electron. Syst., 2019.

[37] H. Wymeersch, et al., "Cooperative Localization in Wireless Networks," Proc. IEEE, vol. 97, 2009.

[38] S. Zhang, et al., "Self-Aware Swarm Navigation in Autonomous Exploration Missions," (accepted).

[39] M. Viberg and A. L. Swindlehurst, "A Bayesian approach to autocalibration for parametric array signal processing," IEEE Transactions on Signal Processing, vol. 42, 1994.

[40] R. Giubilato, et al., "Relocalization With Submaps: Multi-Session Mapping for Planetary Rovers Equipped With Stereo Cameras," Robotics and Automation Letters (RA-L), vol. 5, 2020.

[41] M. G. Müller, et al., "Efficient Terrain Following for a Micro Aerial Vehicle with Ultra-Wide Stereo Cameras," in IEEE AeroConf, 2020.

[42] J. Bertrand, et al., "Roving on Phobos: Challenges of the MMX Rover for Space Robotics," in ASTRA. 